\title{
Avant-propos de la seconde édition
}

Bien que la pratique de la spectroscopie en RMN ait beaucoup évolué ces 20 dernières années, les principes physiques qui sous-tendent la RMN d'états liquides - à la fois objet et objectif de ce petit livre sont restés, pour l'essentiel, inchangés. En 1995, nous avions déjà une excellente compréhension des déplacements chimiques, des couplages scalaires ou "spin-spin ", des échanges chimiques, de la relaxation de spin et des effets produits au niveau des spectres de RMN, et cela était vrai du moins au niveau de la plupart des cours universitaires donnés en licence (ou équivalents). Par conséquent, le contenu de cette seconde édition ne diffère pas énormément de celui de la première. Les ajouts les plus significatifs au texte visent à décrire des techniques expérimentales plus proches de nous, par exemple INEPT, HSQC et la RMN tridimensionnelle.

J'ai corrigé quelques erreurs, quelques imprécisions et certaines présentations par trop simplifiées de la première édition, sans - du moins, je l'espère - en introduire de nouvelles. Si jamais vous vous rendez compte d'une anomalie, quelle qu'elle soit, s'il vous plaît, faites-le moi savoir. Rétrospectivement, quelques sections de la première édition ne me semblaient plus satisfaisantes. En particulier, j'ai réarrangé les sections sur l'anisotropie de groupes voisins, sur le couplage fort, les spins équivalents, le couplage dipolaire, sur le modèle vectoriel et sur la RMN à deux dimensions. Les symboles utilisés pour les fréquences de Larmor et les rotations induites par des impulsions radiofréquences sont maintenant conformes aux usages modernes. J'ai également apporté de nombreux petits changements au texte pour le rendre plus facile à suivre, et quelques améliorations « cosmétiques » aux figures.

J'ai ajouté dix exercices à la fin de chaque chapitre. Certains sont plutôt simples à résoudre, d'autres sans doute présentent un défi aux lecteurs. Mon espoir est qu'ils vous aident à valider et à approfondir votre compréhension des principes de la RMN. Les réponses aux problèmes sont données à la fin du livre. Chaque chapitre se termine par un bref résumé.

J'ai rédigé - coécrit avec Jonathan Jones et Steve Wimperis en 2000 un autre livre chez Oxford University Press, dans la série Introduction à la chimie, sous le titre, NMR: the Toolkit [boîte à outils], qui va aussi connaître sa seconde édition. II peut être considéré comme la suite logique de celui-ci, et s'adresse à tous ceux qui souhaitent en savoir plus sur le fonctionnement opérationnel des séquences de pulsation de RMN et se faire une idée plus précise quant aux mécanismes de la mécanique quantique sous-jacents. Ainsi, les lecteurs qui seraient peutêtre frustrés par une absence de détail et de traitement rigoureux sur 
ces questions quantiques pourraient trouver The Toolkit plus en phase avec leurs goûts personnels.

Je tiens à exprimer ma gratitude à Rod Wasylishen pour le temps passé à identifier, comme il l'a fait, certaines lacunes de la première édition. Je remercie aussi beaucoup Geoffrey Bodenhausen et Gareth Morris qui m'ont gentiment transmis des commentaires détaillés sur le texte de la première édition et de sages suggestions en vue d'y apporter des améliorations. Je remercie également Susannah Worster pour son aide sur le glossaire, Jakub Sowa pour son aide avec Chemdraw ${ }^{\circledR}$, Christina Redfield pour leurs spectres de $950 \mathrm{MHz}$ du lysozyme et Pete Biggs qui a réussi à redonner vie à des versions sur ordinateur de figures que j'avais dessinées il y a 20 ans mais au moyen d'un logiciel aujourd'hui obsolète.

Oxford, octobre 2014, P. J. Hore 
